\title{
Barrier Function
}

1. The placenta is an imperfect barrier (allows many substances to cross)

2. Cytochrome P-450 isoenzymes are located in the placenta, and some may be inducible

(a) These enzymes may metabolize agents and decrease fetal exposure

3. Placenta may also bind substances to minimize fetal exposure to and accumulation of substances 